PPPL-3144 - Preprint: October 1995, UC-420, 426, 427

\title{
3D Fokker-Plank Modeling of Axisymmetric Collisional Losses of Fusion Products in TFTR
}

\author{
V. Ya. Golorborod'ko et al. \\ Princeton Plasma Physics Laboratory \\ P.O. Box 451 \\ Princeton, NJ 08543
}

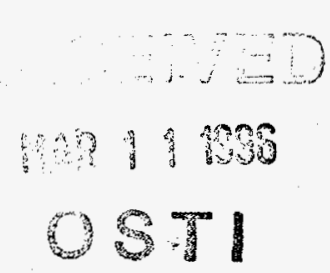

\section{DISCLAIMER}

\begin{abstract}
This report was prepared as an account of work sponsored by an agency of the United States Government. Neither the United States Government nor any agency thereof, nor any of their employees, makes any warranty, express or implied, or assumes any legal liability or responsibility for the accuracy, completeness, or usefulness of any information, apparatus, product, or process disclosed, or represents that its use would not infringe privately owned rights. Reference herein to any specific commercial product; process, or service by trade name, trademark, manufacturer, or otherwise does not necessarily constitute or imply its endorsement, recommendation, or favoring by the United States Government or any agency thereof. The views and opinions of authors expressed herein do not necessarily state or reflect those of the United States Government or any agency thereof.
\end{abstract}

\section{MASIER}


4 and 


\section{NOTICE}

Page(s) size did not permit electronic reproduction. Information may be purchased by the general public from the National Technical Information Service, U.S. Department of Commerce, Springfield, VA 22161 (Area Code 703-487-4650). DOE and DOE contractors may purchase information by contacting DOE's Office of Scientific and Technical Information, P.O. Box 62, Oak Ridge, TN 37831, Attn: Information Services (Area Code 423-576-8401). 


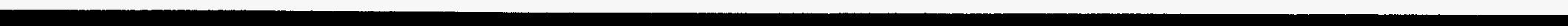




\section{PPPL-3144, Preprint: October 1995}

3D Fokker-Planck Modeling of Axisymmetric Collisional Losses of Fusion Products in TFTR

V. Ya. Golorborod'ko, S.N. Reznik, V.A. Yavorskij, and S.J. Zweben

Results of a 3D (in constants of motion space) Fokker-Planck simulation of collisional losses of fusion products in axisymmetric DT and DD discharges on TFTR are presented. The distributions of escaped ions over poloidal angle, pitch angle, and their energy spectra are obtained. Axisymmetric collisional losses of fusion products are found to be less than (2-5)\%. The distribution of confined fusion products is shown to be strongly anisotropic and nonuniform in the radial coordinate mainly for slowed-down fusion products with small longitudinal energy. Comparison of these results of modeling and experimental data is done. 


\title{
3D Fokker-Planck modeling of axisymmetric collisional losses of fusion products in TFTR
}

\author{
V.Ya. Goloborod'ko, S.N. Reznik, V.A. Yavorskij \\ Institute for Nuclear Research of the National Academy of Sciences of Ukraine, Kiev, \\ Ukraine \\ S.J. Zweben \\ Princeton Plasma Physics Laboratory, P.O. Box 451, Princeton, NJ 08543 USA
}

\begin{abstract}
Results of a 3D (in constants of motion space) Fokker-Planck simulation of collisional losses of fusion products in axisymmetric DT and DD discharges on TFTR are presented. The distributions of escaped ions over poloidal angle, pitch angle, and their energy spectra are obtained. Axisymmetric collisional losses of fusion products are found to be less than $(2-5) \%$. The distribution of confined fusion products is shown to be strongly anisotropic and nonuniform in the radial coordinate mainly for slowed-down fusion products with small longitudinal energy. Comparison of these results of modeling and experimental data is done.
\end{abstract}

\section{Introduction}

Successful experiments on the Tokamak Fusion Test Reactor [1] with DD and DT plasmas gave rise to a number of numerical investigations of the behavior of fusion products (FP) in this device [2-5]. One of the main purposes of such simulations is to determine the inevitable loss level, i.e., to evaluate first-orbit loss [2,3] and ripple-induced stochastic loss [4] which form the minimum losses in the absence of anomalous loss processes [5]. However, collisional losses caused mainly by pitch-angle scattering of fast FP into first-orbit loss cone 
can increase background level even in axisymmetric low (or moderate) current tokamak configuration [6-8].

The main goal of this paper is 3D Fokker-Planck modeling of collisional losses of FP in TFTR DD and DT discharges in axisymmetric limit. It should be pointed out that results of Refs. $[6,8]$ cannot be directly applied to the TFTR experimental results on FP loss because the real experimental conditions were not taken into account in Refs. [6,8]. Specifically, modeling of Refs. [6,8] was carried out for the case without a gap between the plasma and chamber walls, and for low current plasmas $(\mathrm{I}<1.5 \mathrm{MA})$ when none of fattest bananas are confined. On the other hand, real TFTR discharges are characterized by extremely peaked radial profiles of FP source and strong magnetic shear, which in the presence of the gap requires a very careful description of magnetic configuration (including flux surface shift and high-order toroidicity effects).

\section{The Model Used}

\subsection{Model magnetic field}

We will approximate the TFTR magnetic field by a model field with an axisymmetric magnetic toroidal configuration with nested circular flux surfaces of the following form

$$
\overrightarrow{\mathrm{B}}=\frac{\nabla \phi \times \nabla \chi}{\chi^{\prime}(\phi, \chi)}-\frac{\nabla \phi \times \nabla \varphi}{\mathrm{q}(\phi)}
$$

Here, the flux coordinates are $\{\phi, \chi, \varphi\}$ with $\phi$ being toroidal flux, $\chi$ and $\varphi$ being poloidal and toroidal angles, and $q(\phi)$ is the safety factor. For $\varphi$ we will use the angular coordinate of the cylindrical coordinate system $\{r, \varphi, z\}$ (see Fig. 1). Thus, for toroidal components of $\overrightarrow{\mathrm{B}}$ we have [9]

$$
\overrightarrow{\mathrm{B}}_{\mathrm{t}}=\mathrm{F} \nabla \varphi
$$


where $F$ is the poloidal current outside the flux surface (divided by $\mathrm{c} / 2=1.5 \cdot 10^{10} \mathrm{~cm} \cdot \mathrm{s}^{-1}$ ). As an angular coordinate, we will use $\chi$ defined in the following way

$$
\begin{aligned}
& r=r_{0}(\phi)+r_{1}(\phi) \cos \chi, \\
& z=r_{1}(\phi) \sin \chi,
\end{aligned}
$$

where $r_{1}(\phi)$ and $r_{0}(\phi)$ are the minor radius and major radial position of the geometrical center of the flux surface with toroidal flux $\phi$. We introduce also radial shift $\Delta(\phi)$ of the flux surface as follows Ref. [10]

$$
\Delta(\phi)=\mathrm{r}_{0}(\phi)-\mathrm{R}
$$

where $R=r_{0}\left(\phi_{a}\right)$ is the distance from axis of symmetry to the geometrical center of the flux surface corresponding to plasma boundary $\left(\phi=\phi_{a}\right)$. Then for $\chi$ we obtain [11]

$$
\chi^{\prime}=H\left(\varepsilon, \Delta^{\prime}\right) \frac{1+\varepsilon \cos \chi}{1+\Delta^{\prime} \cos \chi}, \quad H\left(\varepsilon, \Delta^{\prime}\right)=\frac{\varepsilon-\Delta^{\prime}\left(1-\sqrt{1-\varepsilon^{2}}\right)}{\varepsilon \sqrt{1-\varepsilon^{2}}}
$$

Here, $\varepsilon=\mathrm{r}_{1}(\phi) / \mathrm{r}_{0}(\phi)$ is flux surface inverse aspect ratio, $\Delta^{\prime}=\mathrm{dr}_{0} / \mathrm{dr}_{1}$.

$$
\mathrm{x}=\frac{\mathrm{r}_{1}(\phi)}{\mathrm{a}}
$$

where $a=r_{1}\left(\phi_{a}\right)$ is the plasma radius. Then, using the numerical results of $[12,13]$, the safety factor and flux surface shift in the plasma can be approximated as follows

$$
q(x)=q_{0}+\left(q_{a}-q_{0}\right) x^{2}
$$




$$
\Delta(\mathrm{x})=\Delta_{0}\left(1-\mathrm{x}^{2}\right)
$$

Resulting profiles of $\mathrm{q}(\mathrm{x})$ and $\Delta(\mathrm{x})$ in the gap $(\mathrm{x}>1)$ are given in Fig. 2. One can see that at $x>1$ profiles of $\mathrm{q}(\mathrm{x})$ deviate sufficiently from that in the quasicylindric approximation $\left(q(x)=q(1) x^{2}\right)$. Typical distributions of the current over the cross section $(\varphi=c o n s t)$ corresponding to $q(x)$ and $\Delta(x)$ of Fig. 2 are given in Fig. 3. It should be pointed out that

$$
\left|\mathrm{j}_{\mathrm{t}}(\mathrm{x}>1)\right| \leq 10^{-2}\left|\mathrm{j}_{\mathrm{t}}(\mathrm{x}<1)\right|
$$

Thus, the profiles of $q(x)$ and $\Delta(x)$ at $x>1$ really correspond to a "currentless" gap.

\subsection{Plasma parameters}

For approximation of the basic plasma parameters (plasma density, ion and electron temperatures), we use the following expressions

$$
\begin{aligned}
& n(x)=\left\{\begin{array}{c}
\left(n_{0}-n_{a}\right)\left[0.75\left(1-x^{2}\right)^{6}+0.25\left(1-x^{2}\right)^{0.3}\right]+n_{a}, \quad x<1 \\
n_{a}, \quad x>1
\end{array},\right. \\
& T(x)=\left\{\begin{array}{cr}
\left(T_{0}-T_{a}\right)\left(1-x^{2}\right)^{2}+T_{a}, & x<1 \\
T_{a} & x>1
\end{array}\right.
\end{aligned}
$$

which are in good agreement with results of Ref. [12]. For FP source profiles $S_{0}(r)$, we use those obtained in Ref. [13]. The doppler broadening of the FP source over V is approximated as follows [14]

$$
\mathrm{S}\left(\mathrm{r}, \frac{\mathrm{v}}{\mathrm{v}_{\mathrm{o}}}\right)=\frac{\mathrm{S}_{\mathrm{o}}(\mathrm{r})}{4 \pi \mathrm{v}_{0}^{3}} \sqrt{\gamma / \pi} \frac{\mathrm{v}_{0}}{\mathrm{v}} \exp \left(-\gamma\left(\frac{\mathrm{v}}{\mathrm{v}_{\mathrm{O}}}-1\right)^{2}\right), \quad \gamma=\frac{\mathrm{E}_{\mathrm{O}}}{\mathrm{T}_{\mathrm{i}}}\left\{\begin{array}{l}
1.25, \text { alphas } \\
1.33, \text { tritons }
\end{array}\right.
$$


All the rest of the required parameters are given in Table 1.

\section{Basic Equations}

To describe collisional behavior of nonthermalized FPs, one can proceed from the following bounce-averaged Fokker-Planck equation in 3D COM space [15-18]

$$
\frac{\partial f}{\partial t}+L f=S(\vec{c})
$$

Here, $L$ and $S$ are orbit averaged drift Fokker-Planck operator and fast particle source, respectively. We will use $3 \mathrm{D}$ COM space $\{\overrightarrow{\mathrm{c}}\} \equiv\left\{\mathrm{c}^{1}, \mathrm{c}^{2}, \mathrm{c}^{3}\right\}$ with $[15,18]$

$$
c^{1}=v, c^{2}=\lambda, c^{3}=\phi_{m}
$$

In Eq. (14) $v$ is the particle velocity; $\lambda=\left(v_{\perp}^{2} B_{0}\right) /\left(v^{2} B\right)$ is the transverse adiabatic invariant; $B_{0}$ is the magnetic field at magnetic axis; and $\phi_{m}$ is the maximum value of radial coordinate along the guiding-center orbit [19]. Orbit averaging in this paper is determined as follows [18]

$$
\langle F\rangle=\frac{1}{\sqrt{g_{\vec{c}}}} \oint d \theta \sqrt{g_{\vec{c}}} F, \sqrt{g_{\vec{c}}}=\oint d \theta \sqrt{g}
$$

where $\theta$ is cyclic variable, defined by

$$
\sin ^{2} \theta=\left(\phi_{\mathrm{m}}-\phi\right) / \Delta \phi
$$

with $\phi$ being the radial flux coordinate (toroidal magnetic flux), $\Delta \phi=\phi_{m}-\phi_{\min }, \phi_{\min }$ is minimum value of $\phi$ along orbit; $\sqrt{g}$ is Jacobean for following 4D space 


$$
\{\vec{x}\} \equiv\left\{x^{1}, x^{2}, x^{3}, x^{4}\right\}=\left\{c^{1}, c^{2}, c^{3}, \theta\right\} .
$$

The averaged Fokker-Planck operator (see [18]) can be represented as

$$
L=\frac{1}{\sqrt{g_{\vec{c}}}} \sum_{k, l=1}^{3} \frac{\partial}{\partial c^{k}} \sqrt{g_{\vec{c}}}\left(\hat{d}^{k}-\hat{D}^{k l} \frac{\partial}{\partial c^{l}}\right)
$$

with the following orbit averaged transport coefficients

$$
\begin{aligned}
& \hat{d}^{k}=\left\langle d_{\vec{x}}^{k}(\theta)-D_{\vec{x}}^{k} 4 \underset{0}{\theta}(\theta) d \theta^{\prime} H\left(\theta^{\prime}, \theta\right) \frac{\partial}{\partial \theta}\left[\sqrt{g_{\vec{x}}\left(\theta^{\prime}\right)} d \frac{4}{\vec{x}}\left(\theta^{\prime}\right)\right]\right\rangle \\
& \hat{D}^{\mathrm{kl}}=\left\langle\mathrm{D}_{\overrightarrow{\mathrm{x}}}^{\mathrm{kl}}(\theta)\right\rangle- \\
& \left\langle\int_{0}^{\theta} \mathrm{d} \theta^{\prime} \mathrm{H} \frac{\partial}{2 \cdot \partial \theta^{\prime}}\left[\sqrt{g_{\overrightarrow{\mathrm{x}}}}\left(D_{\overrightarrow{\mathrm{x}}}^{\mathrm{k} 4}(\theta) \mathrm{D}_{\overrightarrow{\mathrm{x}}}^{14}\left(\theta^{\prime}\right)+\mathrm{D}_{\overrightarrow{\mathrm{x}}}^{\mathrm{k} 4}\left(\theta^{\prime}\right) \mathrm{D}_{\overrightarrow{\mathrm{x}}}^{14}(\theta)\right)\right]\right\rangle
\end{aligned}
$$

In Eqs. (19) and (20) $d_{\overrightarrow{\mathrm{x}}}^{k}$ and $D_{\overrightarrow{\mathrm{x}}}^{\mathrm{kl}}$ are contravariant component of vector of convection and tensor of diffusion in $\{\bar{x}\}$ space

$$
\begin{aligned}
& d_{\vec{x}}^{k}=-v_{s} v \frac{\partial x^{k}}{\partial V} ; D_{\vec{x}}^{k l}=v_{\perp}\left(1-\xi^{2}\right) \frac{\partial x^{k}}{\partial \xi} \frac{\partial x^{1}}{\partial \xi} \\
& \sqrt{g}=\frac{v^{2}\left|\partial J / \partial \phi_{m}\right| \Delta \phi}{2|\nabla \varphi|^{2} F^{2} \rho_{L}} \frac{b^{3}|\sin \theta|}{(2-\lambda b)\left|b_{\chi}\right| \chi^{\prime}} .
\end{aligned}
$$

Here

$$
\frac{\partial x^{1}}{\partial V}=1 ; \frac{\partial x^{2}}{\partial V}=\frac{\partial x^{1}}{\partial \xi}=0 ; \frac{\partial x^{2}}{\partial \xi}=-\frac{2 \xi}{b}
$$




$$
\begin{aligned}
& \frac{\partial \mathrm{x}^{4}}{\partial \mathrm{z}}=\frac{2}{\Delta \phi \sin \theta}\left(\frac{\partial \phi_{\mathrm{m}}}{\partial \mathrm{z}} \cos ^{2} \frac{\theta}{2}+\frac{\partial \phi_{\min }}{\partial \mathrm{z}} \sin ^{2} \frac{\theta}{2}\right) \\
& \frac{\partial \phi_{\alpha}}{\partial \mathrm{V}}=\frac{\Psi_{\alpha}-\psi(\phi)}{\mathrm{V} \partial \mathrm{J} / \partial \phi_{\alpha}} ; \frac{\partial \phi_{\alpha}}{\partial \xi}=\rho_{L} \frac{\mathrm{F}_{\alpha} \xi / \xi_{\alpha}-\mathrm{F}}{\mathrm{b} \partial \mathrm{J} / \partial \phi_{\alpha}} ; \alpha=(\mathrm{m}, \min ) ; \mathrm{z}=\mathrm{v}, \xi
\end{aligned}
$$

and

$$
\frac{\partial J}{\partial \phi_{\alpha}}=\left(\frac{1}{q}-F \rho_{L} \frac{1+\xi^{2}}{2 \xi} \frac{\partial b^{-1}}{\partial \phi}-\frac{\rho_{L} \xi}{b} \frac{\partial F}{\partial \phi}\right)_{\alpha}, \rho_{L}=\frac{m c V}{e_{c}}
$$

For characteristic collisional frequencies of slowing-down $\left(v_{s}\right)$ and pitch-angle scattering $\left(v_{\perp}\right)$, we will use following expressions (see [20])

$$
\begin{aligned}
& v_{\perp}=\Sigma\left[\mu\left(x_{\beta}\right)\left(1-0.5 x_{\beta}^{-1}\right)+\mu^{\prime}\left(x_{\beta}\right)\right] v_{0 \beta} \\
& v_{S}=2 \Sigma\left(m / m_{\beta}\right) \mu\left(x_{\beta}\right) v_{0 \beta}
\end{aligned}
$$

where

$$
\begin{aligned}
& v_{o \beta}=2 \pi e^{2} e_{\beta}^{2} \lambda_{\beta} n_{\beta} /\left(m^{2} v^{3}\right), x_{\beta}=\frac{m_{\beta} v^{2}}{2 T_{\beta}} \\
& \mu(x)=\frac{2}{\sqrt{\pi}} \int_{0}^{x} d t \sqrt{t} \exp (-t) .
\end{aligned}
$$

Note that in the Fokker-Planck operator, we neglect parallel diffusion for fast ions since $v_{\mathrm{ll}} \propto \mathrm{T} / \mathrm{E}$ and is small compared to $v_{s}$ and $v_{\perp}$. To complete the formulation of the boundary value problems for the FP distribution function, one should find the definition domain in COM space and supply Eq. (10) with boundary conditions. Definition domains 
for $3.5 \mathrm{MeV}$ alphas on $\left\{\lambda, \mathrm{r}_{\mathrm{m}}\right\}$ plane are given in Fig. 4. They correspond to TFTR DT discharge with $I=1.0 \mathrm{MA}$. In Fig. 5 are shown the orbit averaged alpha-particle source for this case.

\section{Results of Simulation}

\subsection{Classical losses of alphas and tritons}

First we consider classical losses due to the prompt or first-orbit loss mechanism. The prompt loss fraction of alphas as a function of plasma current for the parameters listed in Table 1 is shown in Fig. $6 \mathrm{a}$. Figure $6 \mathrm{~b}$ shows the poloidal distributions of the first-orbit loss for different currents. Note that these distributions are significantly changed as the current increases. Therefore, dependencies of first-orbit losses detected at fixed poloidal angles on plasma current differ from that for the total prompt loss. This is confirmed by Fig. $7 \mathrm{a}$ where dependencies of the local prompt losses corresponding to $\chi=20^{\circ}, 45^{\circ}, 60^{\circ}$ and $90^{\circ}$ on I are given (to take into account detector aperture, only particles detecting in $45^{\circ}-83^{\circ}$ pitch angle range were considered).

Figure 6a also shows collisional losses of FPs in energy range $0.25<E / E_{0}$ (here, $\mathrm{E}_{0}$ $=3.5 \mathrm{MeV}$ for alphas and $E_{0}=1.01 \mathrm{MeV}$ for tritons) versus plasma current. One can see that maximum collisional loss fraction takes place at I $<1$ MA and decreases with increasing current (when I $>1 \mathrm{MA}$ ). Figure $6 \mathrm{c}$ shows the distribution of collisionally lost alphas versus poloidal angle. Note that in the cases of I $>1.4 \mathrm{MA}$ and $\mathrm{I}<1 \mathrm{MA}$ the maximum loss takes place at poloidal angles $\chi>90^{\circ}$, i.e., the poloidal distributions of first-orbit and collisional losses are qualitatively different. Dependencies of collisionally lost alphas detected at poloidal angles $\chi=20^{\circ}, 45^{\circ}, 60^{\circ}$ and $90^{\circ}$ on plasma current are shown in Fig. $7 \mathrm{~b}$. Comparing Figs. $6 \mathrm{c}$ and $7 \mathrm{~b}$ one can conclude that collisional losses of alphas in axisymmetric TFTR discharges are small compared to first-orbit losses. Only at small poloidal angles are both kind of losses comparable to each other. Results are given in Fig. 6d for classical collisional losses of tritons in axisymmetric DD discharges in TFTR for the parameters of 
Table 1. It should be pointed out that the collisional losses of tritons calculated in this paper are several times smaller than that obtained in Ref. [8] for small current axisymmetric tokamaks. The main reason of decreasing of losses are the existence of the gap between plasma and wall, the strong magnetic shear, and the strongly peaked source profile in contrast to the discharge parameters chosen in Ref. [8].

In spite of the low level of axisymmetric collisional losses, they may affect the total pitch angle distribution of escaped FPs. This is caused by the strong localization of collisionally lost particles over $\mathrm{V}_{\mathbb{N}} / \mathrm{V}$ as shown in Fig. 8, where the pitch angle distributions of collisional and prompt losses of tritons at $90^{\circ}$ poloidal angle are presented. One can see that in the pitch angle range $62^{\circ}-68^{\circ}$ collisional losses dominate over prompt ones. In the case of alphas, the influence of collisionally lost particles on the total pitch angle distribution is weaker.

\subsection{Distribution function of confined alphas}

Figure 9 shows the distribution function of fusion alpha particles for an axisymmetric TFTR DT discharge with $\mathrm{I}=1.0 \mathrm{MA}$ at different energies. Comparing the initial distribution (Fig. 5) and that of $\mathrm{E} / \mathrm{E}_{\mathrm{O}}=0.56$ and $\mathrm{E} / \mathrm{E}_{\mathrm{O}}=0.25$ (see Fig. 9), one can conclude that the collisional evolution of fast alphas increases the population of co-moving particles with low longitudinal energy in the outboard plasma region. To clarify this effect, we consider the distribution of confined alphas in Eulerian velocity coordinates. Figure 10 represents pitch angle dependence of the distribution function in the velocity range $0.5<\mathrm{V} / \mathrm{V}_{\mathrm{o}}<1\left(\mathrm{~V}_{\mathrm{o}}=1.3\right.$ $\times 10^{9} \mathrm{~cm} \mathrm{~s}^{-1}$ ) for different values of $r$ on the equatorial plane. Figures $10 \mathrm{a}$ and $10 \mathrm{~b}$ correspond, respectively, to the outer and inner parts of the flux surface with $r_{1} / a=0.5$. One can see that the distribution function is essentially anisotropic in the considered velocity range. Figure 10a shows that the population of particles with small longitudinal energy (IV${ }_{\|} / \mathrm{VI}<0.1$ ) at the outer part of the plasma column is increased most rapidly in the process of slowing down (for pitch angles of about $90^{\circ}$ the ratio $f\left(\mathrm{~V}=0.5 \mathrm{~V}_{\mathrm{o}}\right) / f\left(\mathrm{~V}=\mathrm{V}_{\mathrm{o}}\right.$ ) is about 20 , 
while for other pitch angles this ratio is at least two times less). The reason for this increase is the enhanced outward radial shift of the bi-axial orbits with $V_{\|} \approx 0$ due to slowing down compared to rest of these particles. This conclusion is confirmed by Fig. 10c, where the distribution function of alphas on the magnetic axis of plasma is shown. One can see that the number of particles with pitch angles in the range $80^{\circ}-100^{\circ}$ is increased by only three times when the velocity decreases from $V=V_{0}$ to $V=0.5 V_{0}$ while, for the rest particle pitch angles, corresponding increase is about four times. The effect of the outward radial shift of slowed down particles with small longitudinal velocity is demonstrated also by Fig. $11 \mathrm{~b}$, where the radial profiles of a trapped particle density with $\mathrm{E}=3.5 \mathrm{MeV}$ and $\mathrm{E}=2 \mathrm{MeV}$ are shown.

The anisotropy of confined alpha distribution results in longitudinal current. The radial profile of density of this current is shown in Fig. 11a.

\section{COMPARISON WITH EXPERIMENT}

The TFTR DT experimental results which motivated these calculations are summarized in Ref. 21. The parameters for these discharges are similar to those shown in Table 1, e.g. $\mathrm{I}=0.6-2.0 \mathrm{MA}$ and $\mathrm{R}=2.52 \mathrm{~m}$.

The DD and DT fusion product losses were measured at the detector $90^{\circ}$ below the outer midplane for each of these discharges within the pitch angle range $45-83^{\circ}$. The results were approximately consistent with the calculations presented in Fig. 7, e.g. the measured DT alpha loss decreased by a factor of $4-5$ between 0.6 and $2.0 \mathrm{MA}$, while the calculated loss fraction decreased by a factor of 3.5. This difference could easily be due to the variation in the shapes of the plasma current and neutron source profiles over this current range, which were not taken into account in this calculation.

There was no clear experimental evidence for collisional alpha loss in the $90^{\circ}$ detector in these experiments, i.e. the measured alpha loss energy was consistent with the Dopplerbroadened birth energy, the pitch angle distribution was consistent with the first-orbit loss model, and the time dependence of the alpha loss followed that of the alpha source without a 
delay characteristic of a collisional diffusion [21]. However, the calculated axisymmetric collisional loss component was only $\approx 10 \%$ of the first orbit loss in all these cases, which is well within the experimental uncertainty of all these measurements.

There was an delayed loss component of DD fusion products which has been described previously [5]. However, the pitch angle of this delayed loss was $\approx 70^{\circ}$, i.e. higher than that of the first orbit loss, and not smaller as calculated for collisional triton loss in Fig. 8. However, the data are not inconsistent with the presence of a collisional triton loss component as shown in Fig. 8, given the detector pitch angle resolution of $\pm 5^{\circ}$, and the presence of $3 \mathrm{MeV}$ protons in the detected DD signals.

Measurements have also been made for other detector positions. The data for the plasma current dependence of FP loss at $45^{\circ}$ for these same discharges showed a surprising peak in the DT and DD loss fraction at 1.8 MA [22], which is not predicted by the present calculations shown in Fig. 7. The data for the FP loss at $20^{\circ}$ showed a peak at 1.0 MA [23], which was most likely due to TF ripple loss, and so beyond the scope of the present calculations.

\section{SUMMARY AND DISCUSSIONS}

Simulation of fast $\left(\mathrm{E} / \mathrm{E}_{0}>0.25\right)$ fusion product losses in axisymmetric DD and DT discharges in TFTR show that the fraction of collisionally lost FPs is less than 2\% (for alphas) and $5 \%$ (for tritons), and that this fraction decreases with plasma current above $\mathrm{I}=1.0 \mathrm{MA}$. The main reason for these losses is the pitch angle scattering of fast particles into the "axisymmetric" prompt loss region in velocity space. In the lower energy range $\left(\mathrm{E} / \mathrm{E}_{0}<0.5\right)$, in addition to diffusion collisional loss mechanism, slowed down co-moving FPs passing through the vacuum gap at the plasma edge can be lost due to the decrease of their deviations from flux surface. The maximum axisymmetric collisional flux takes place at poloidal angles $90^{\circ}$ and shifts to regions of higher poloidal angles with increased current. In spite of the 
low level of axisymmetric collisional losses, they may noticeably affect the total pitch angle distribution of the escaped tritons, at least for plasma currents in the order of $1 \mathrm{MA}$.

The pitch angle distribution and energy spectrum of the lost particles is sensitive to the energy distribution of the FP birth source, in contrast to the total FP losses and their poloidal distribution. It is evident that the lost particle energy spectrum is mainly affected by Doppler broadening of the birth source (see Fig. 12).

The distribution of confined FPs is found to be strongly anisotropic, which may induce velocity-space instabilities. Axisymmetric collisional transport of fast fusion products increases the population of slowed down, co-moving particles with small longitudinal velocities in the outward region of the plasma column. This could be important for alphaparticle diagnostics using the pellet charge exchange method and, in the presence of TF ripple, can result in significant additional collisional losses.

\section{Acknowledgments}

This work was supported by the United States Department of Energy, under subcontract S-03691-K and DOE contract No. DE-AC02-76-CHO-3073.

Special recognition is due to A.D. Fursa for providing several subroutines for the computer code. 


\section{Appendix}

Neglecting corrections of order $\left(\mathrm{Aq}_{\mathrm{a}}\right)^{-2} \ll 1$ for $F$ and $\mathrm{B} \equiv|\overrightarrow{\mathrm{B}}|$ we can use

$$
\mathrm{B} \cong \frac{\mathrm{F}}{\mathrm{r}} \cong \frac{\mathrm{B}_{\mathrm{C}}}{\left(1+\frac{\Delta}{\mathrm{R}}\right)(1+\varepsilon \cos \chi)}, \quad \mathrm{F}=\mathrm{RB}_{\mathrm{c}}
$$

here $B_{c}$ is magnetic field at plasma center $(z=0, r=R)$. Thus, for the complete definition of the magnetic configuration in the model used, one should know the safety factor and flux surface shift as functions of the radial coordinate and geometrical parameters of the configuration like $R, A=R / a$ (plasma aspect ratio). Note that the safety factor can be expressed as a ratio of $F$ to the total plasma current (I), the inside flux surface multiplied by a function of only $\varepsilon$ and $\Delta^{\prime}$, i.e., [11]

$$
\begin{aligned}
& q=q_{c} G\left(\varepsilon, \Delta^{\prime}\right) \\
& G\left(\varepsilon, \Delta^{\prime}\right)=\frac{H\left(\varepsilon, \Delta^{\prime}\right)}{\varepsilon-\Delta^{\prime}}\left(\frac{\varepsilon}{\sqrt{1-\varepsilon^{2}}}-\frac{\Delta^{\prime}}{\sqrt{1-\Delta^{2}}}\right)
\end{aligned}
$$

In Eq. (A2),

$$
q_{c}=\frac{c F}{2 A^{2} I}
$$

is a safety factor in the quasicylindrical approximation. For typical TFTR discharges ( $\varepsilon=A^{-1}=0.3 ; \Delta^{\prime} \cong-0.5$ ), the factor $G$ (giving a decrease of rotational transform due to toroidicity [11]) is about 1.3. 
For the definition of $q$ and $\Delta$ in the gap between the plasma and the chamber wall, we assume that the toroidal current $\left(\mathrm{j}_{\mathrm{t}}=\frac{\overrightarrow{\mathrm{j}} \nabla \varphi}{|\nabla \varphi|}\right)$ in the gap is absent. Now, take into account that for the axisymmetric toroidal configuration with the circular flux surfaces [11]

$$
\frac{4 \pi}{\mathrm{c}} \sqrt{\mathrm{g}} \cdot \overrightarrow{\mathrm{j}} \nabla \varphi=\frac{\partial}{\partial \mathrm{x}}\left(\frac{\mathrm{F}}{\mathrm{q}} \mathrm{h}^{2}\right)+\frac{\partial}{\partial \chi}\left(\frac{\mathrm{Fh}{ }^{2} \Delta^{\prime} \sin \chi}{\mathrm{qx}}\right)
$$

where

$$
\mathbf{h}=\frac{\varepsilon H\left(\varepsilon, \Delta^{\prime}\right)}{\left(1+\Delta^{\prime} \cos \chi\right)}
$$

and

$$
\sqrt{g}=\frac{1}{\nabla x \nabla \chi \nabla \varphi}=a r^{2} H
$$

is the Jacobian in the flux coordinate system $\{x, \chi, \varphi\}$. Using Eqs. (13), (14), and (13)-(15), one can conclude that $j_{t}$ is a function of $x, \mathrm{q}(\mathrm{x}), \mathrm{q}^{\prime}(\mathrm{x}), \Delta(\mathrm{x}), \Delta^{\prime}(\mathrm{x}), \Delta^{\prime \prime}(\mathrm{x})$ and $\chi$, i.e.,

$$
\mathrm{j}_{\mathrm{t}}=\mathrm{j}_{\mathrm{t}}(\mathrm{x}, \mathrm{q}(\mathrm{x}), \Delta(\mathrm{x}) ; \chi) .
$$

The dependence of Eq. (16) permits us to formulate a variational problem for definition of $\mathrm{q}(\mathrm{x})$ and $\Delta(\mathrm{x})$ in the gap. For this purpose, we introduce the following integral value

$$
\mathrm{W}[\mathrm{q}, \Delta]=\int_{\Delta \mathrm{S}} \mathrm{dsj} \mathrm{j}^{2}(\mathrm{x}, \mathrm{q}, \Delta ; \chi)
$$

where

$$
\mathrm{ds}=\frac{\mathrm{dxd} \chi|\nabla \varphi|}{\nabla \mathrm{x} \nabla \chi \nabla \varphi}
$$


is an element of the configuration cross section area., $\Delta S$ is the cross section region corresponding to the gap. Using Eq. (17), we can find the $q(x)$ and $\Delta(x)$ that minimize $W$ (or correspond to the "currentless" gap). 


\section{References}

[1] MC GUIRE, K.M., et al., Phys. of Plasmas 2, 2176 (1995).

[2] ZWEBEN, S.J., et al., Nucl. Fusion 31 (1991) 2219.

[3] ZWEBEN, S.J., BUSH, C.E., CHANG, C.S., et al., Phys. Plasmas 1 (1994) 1469.

[4] BOIVIN, R., ZWEBEN, S.J., WHITE, R.B. , Nucl. Fusion 33 (1993) 449.

[5] ZWEBEN, S.J., et al., in Plasma Physics and Controlled Nuclear Fusion Research 1992 (Proc. 14th Int. Conf. Wurzburg, 1992) Paper A-6-3; ZWEBEN, S.J., et al., Nucl. Fusion 31 (1991) 2219.

[6] GOLOBOROD'KO, V.Ya., LUTSENKO, V.V., REZNIK, S.N., YAVORSKIJ, V.A., Collisional Losses of Fast Particles in Axisymmetric Tokamaks, (Proc. IAEA TC Meeting on Alpha Particles in Fusion Research, Trieste, Italy, 1993), IAEA, Vienna (1993) 305.

[7] CHANG, C.S., ZWEBEN, S.J., SCHIVELL, J., BUDNY, R., and SCOTT, S., Phys. Plasmas 1 (1994) 3857.

[8] GOLOBOROD'KO, V.Ya., LUTSENKO, V.V., REZNIK, S.N., YAVORSKIJ, V.A., Collisional losses of fast particles in low current axisymmetric tokamaks, Fusion Technology 27 (1995) 292.

[9] HINTON, F.L., HAZELTINE, R.D., Rev.Mod.Phys. 48 (1976) 239.

[10] ZACHAROV, L.E., SHAFRANOV, V.D., in Problems of Plasma Theory 11 (1982) 118.

[11] YAVORSKIJ, V.A., On axisymmetric toroidal magnetic configurations with elliptic and circular flux surfaces, submitted to Ukr.Phys.J (1995).

[12] BUDNY, R.V., et al., Nucl. Fusion 32 (1992) 429.

[13] BUDNY, R.V., et al., Nucl. Fusion 34, 1247 (1994).

[14] KOLESNICHENKO, Ya.I., ORAEVSKIJ, V.N., At.Ehnerg. 23 (1967) 289.

[15] GOLOBOROD'KO, V.Ya.,YAVORSKIJ, V.A., On 3D Kinetic Approach for Description of Fast Particle Transport in Axisymmetric Tokamak Plasmas, (Proc. 
IAEA TC Meeting on Alpha Particles in Fusion Research, Trieste, Italy, 1993), IAEA, Vienna (1993) 490.

[16] ZAITSEV, F.S., O'BRIEN, M.R., COX, M., Phys. Fluids B 5 (1993) 509.

[17] ERIKSON, L.-G., HELANDER, P., Phys. Plasmas 1 (1994) 308.

[18] GOLOBOROD'KO, V.Ya.,YAVORSKIJ, V.A., On formulation of 3D boundary value problem for fast particle distribution function in axisymmetric tokamak plasmas, submitted to Nuclear Fusion (1995).

[19] ROME, J.A., PENG, Y-K.M., Nucl. Fusion 19 (1979) 1193.

[20] TRUBNIKOV, B.A., in Problems of Plasma Theory, Vol. 1 (LEONTOVICH, M.A., Ed.), Gosatomizdat, Moscow (1963) 98 (in Russian). [English translation: Reviews of Plasma Physics, Vol. 1, Consultants Bureau, New York (1965) 105.]

[21] ZWEBEN, S. J., et al., Alpha Particle Loss in the TFTR DT Experiments, to be published in Nucl. Fusion 1995

[22] ZWEBEN, S.J., et al., Measurements of Escaping Alphas in the TFTR DT Experiments, PPPL-3088, 1995, also Proc. 21st European Physical Society Meeting, Montpellier (1994).

[23] ZWEBEN, S.J., et al., Measurements of Alpha Particle Loss to the Outer Midplane Region of TFTR, to be published in Nuclear Fusion, 1995 
Table I. TFTR parameters

Symbol

Physical Quality

Value

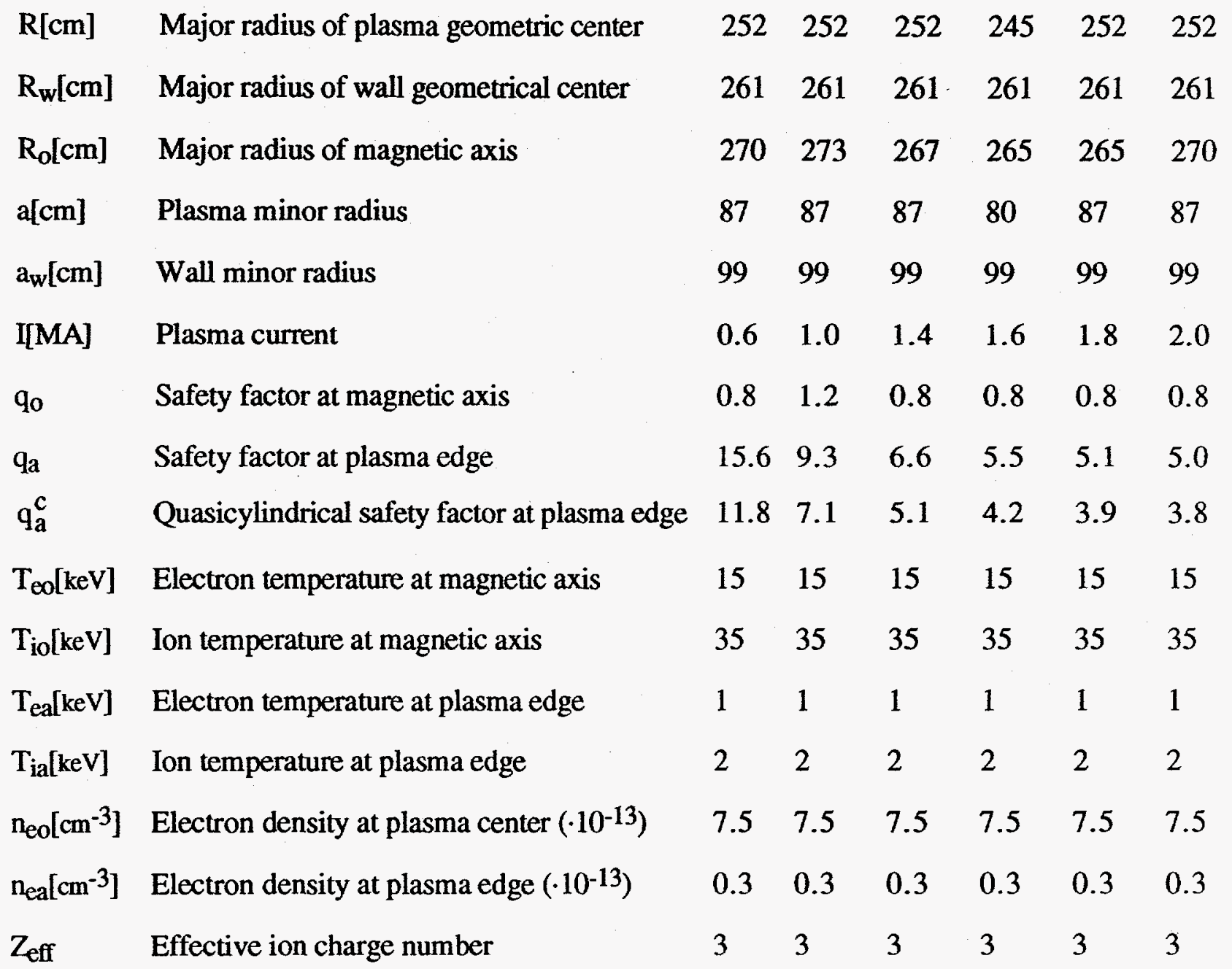




\section{Figure Captions}

Fig. 1. Coordinate system and main geometrical parameters.

Fig. 2. Radial profiles of flux surface shift (divided by plasma radius) and safety factor ( $\mathrm{X}^{2}$ is a toroidal flux, $\mathrm{X}=1$ corresponds to plasma edge).

Fig. 3. Plasma current density corresponding to data of Fig. 2.

Fig. 4. Definition domains for particles with $\sigma=+1$ (a) and $\sigma=-1$ (b). Here $\sigma=$ $V_{\|} / V_{\|} \mid$at $X=X_{m}$.

Fig. 5. Distribution function of alphas at $E=E_{0}$ for $I=1 M A$.

Fig. 6. Classical losses (loss fractions and fluxes) of alphas and tritons.

Fig. 7. Alpha losses detected at different poloidal angles vs. plasma current.

Fig. 8. Pitch angle distributions of lost tritons.

Fig. 9. Distribution function of alphas at different values of $\mathrm{E} / \mathrm{E}_{\mathrm{O}}$ for $\mathrm{I}=1 \mathrm{MA}$.

Fig. 10. Pitch angle distribution of confined alphas at different values of V. Plotted curves are equidistant in $\mathrm{V}$.

Fig. 11. Radial profiles of longitudinal alpha current (a) and density of trapped alpha particles (b). Here $S_{0} \tau_{S o}=S / v_{S}$ for $X=0$ and $V=V_{\alpha}$.

Fig. 12. Energy spectrums of escaped fusion products for monoenergetic and nonmonoenergetic (bold lines) birth sources. 


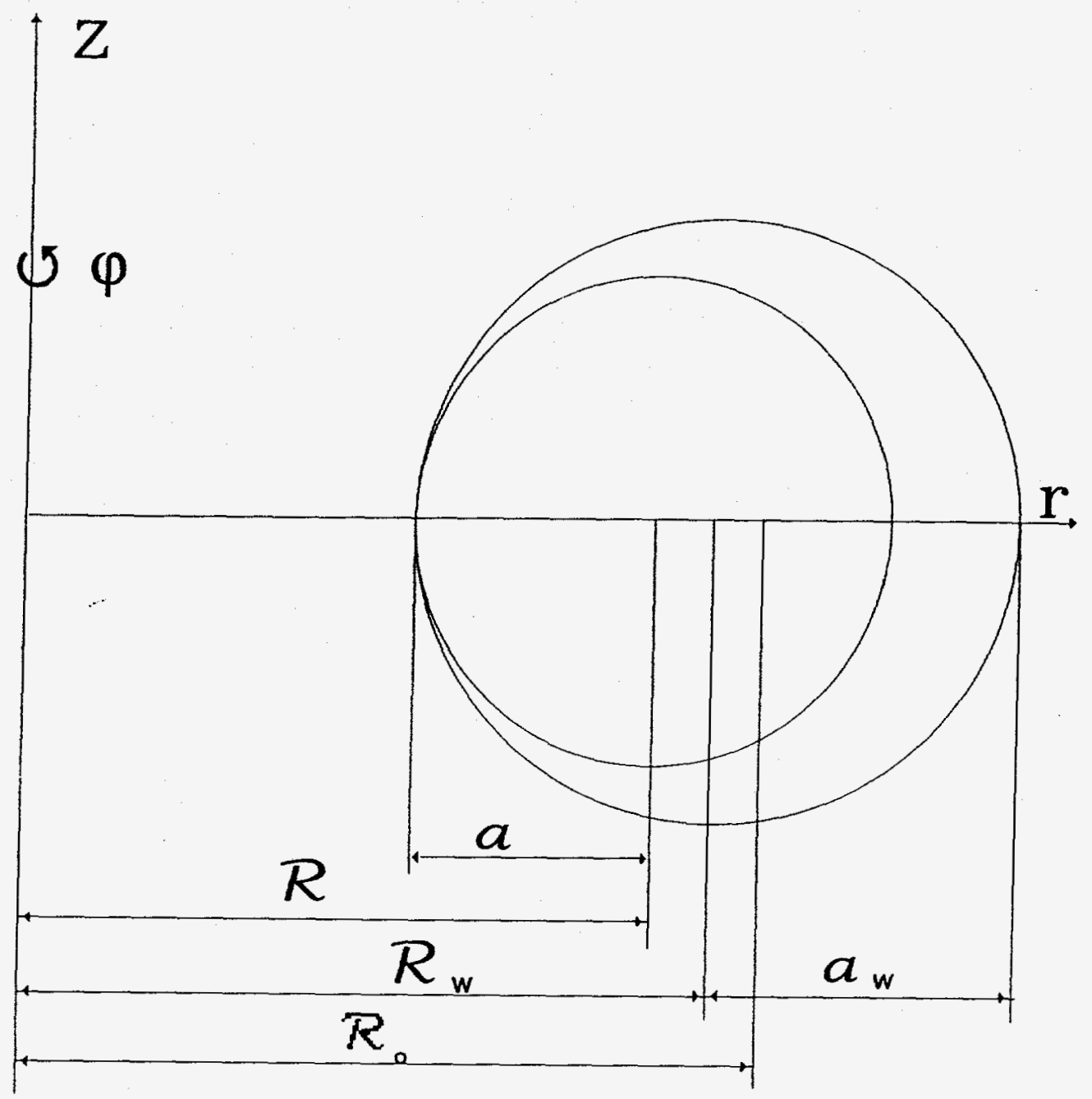

Fig.1 

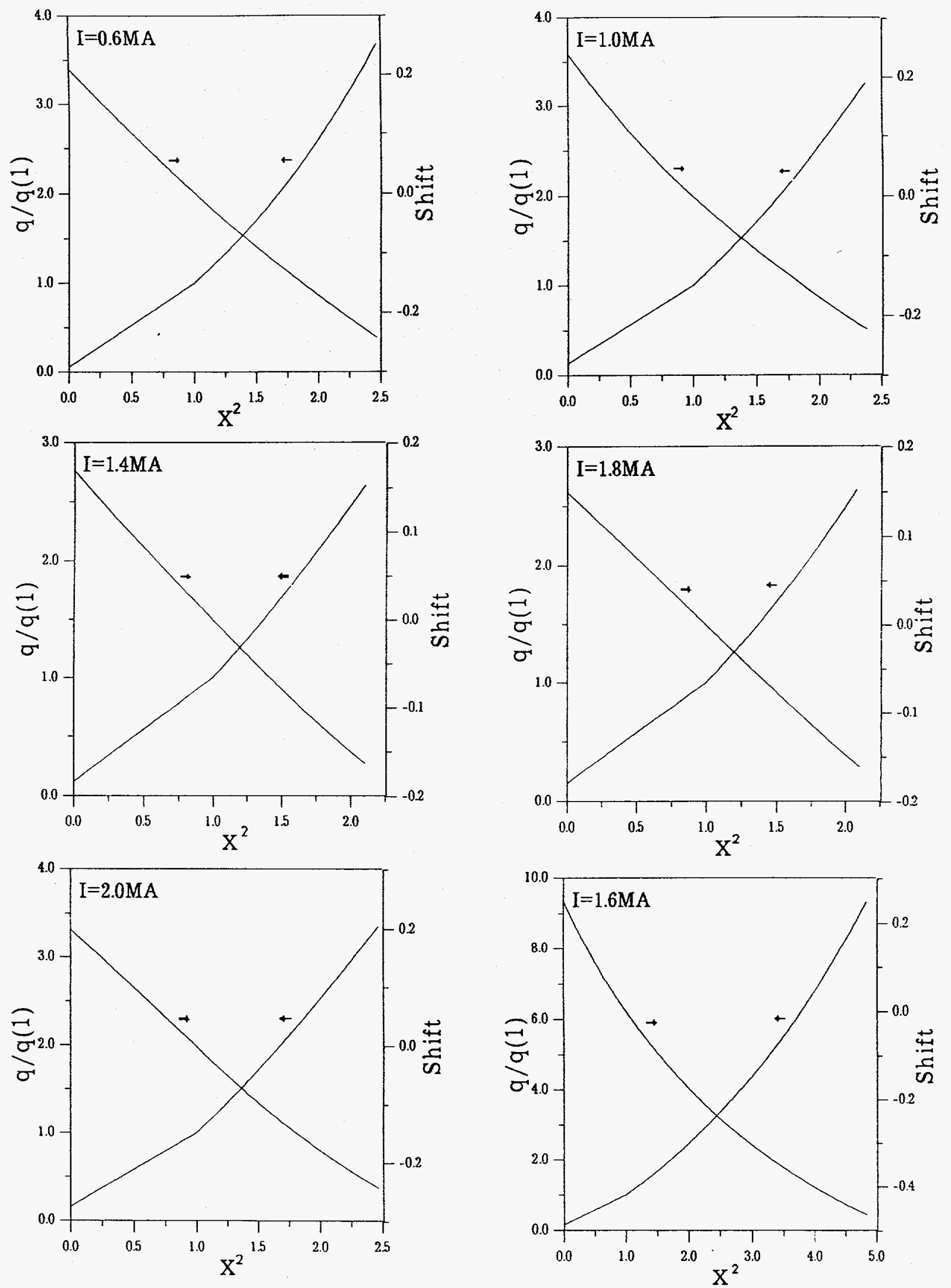

Fig.2 

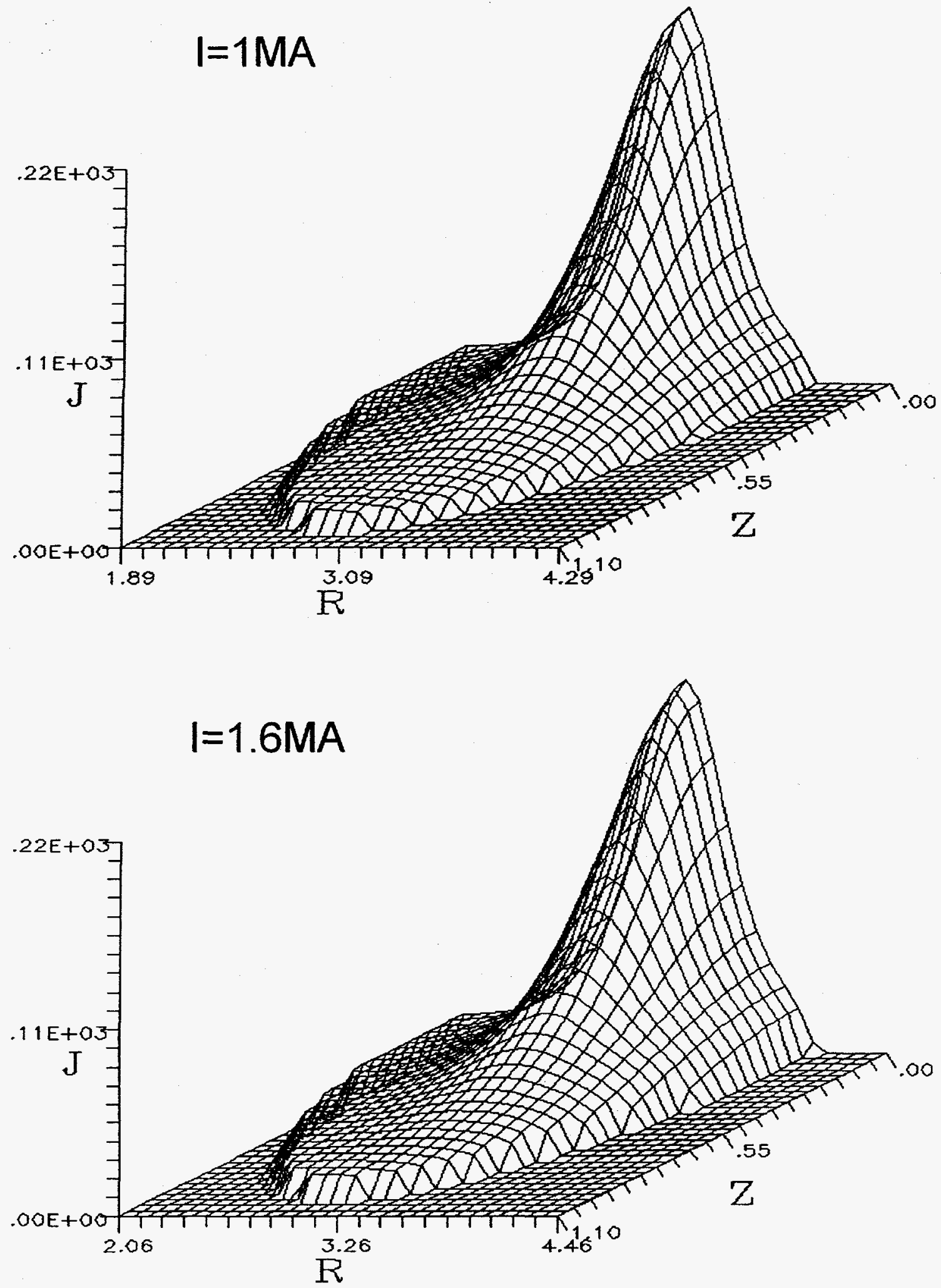

Fig.3 

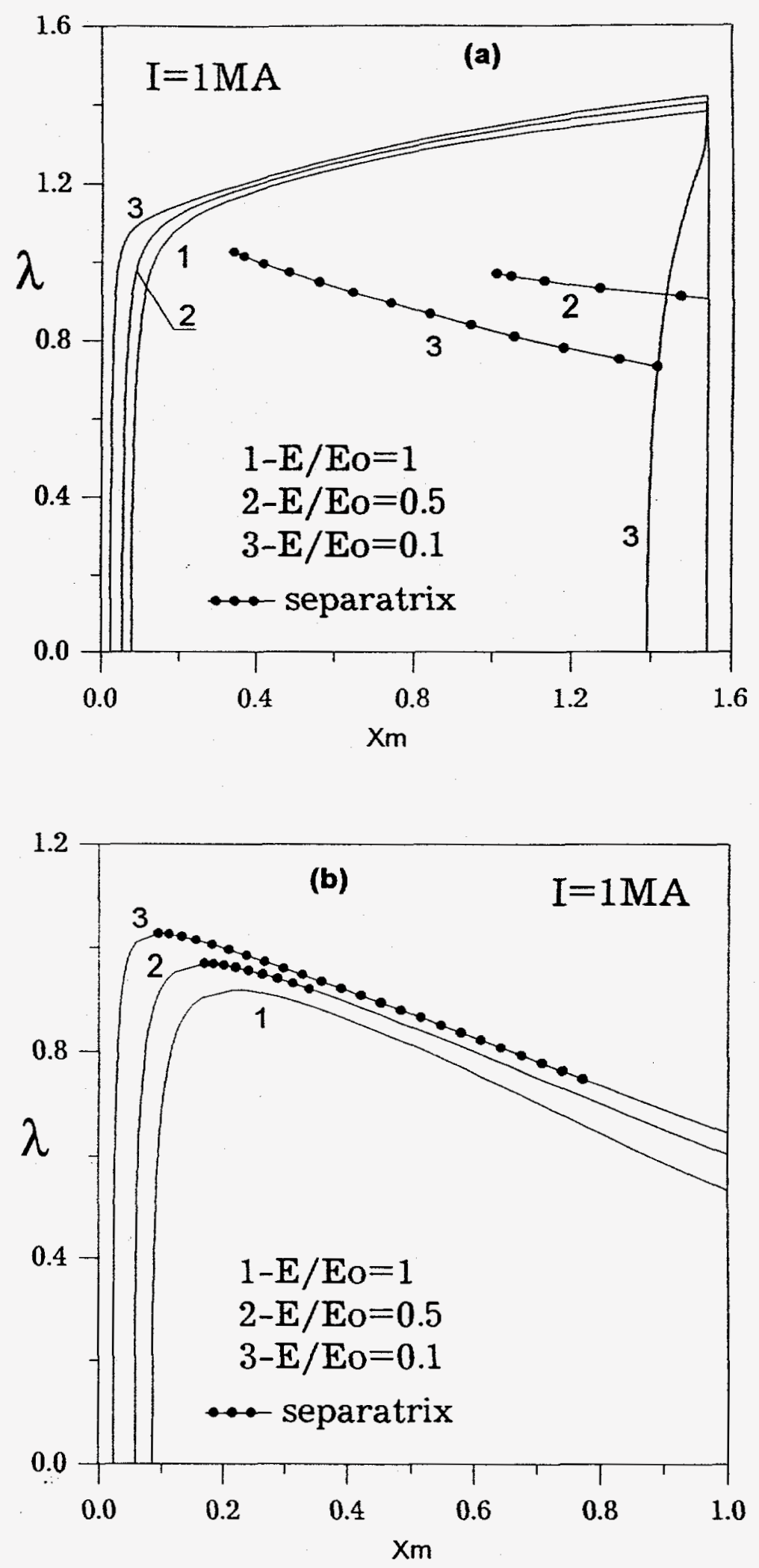

Fig.4 

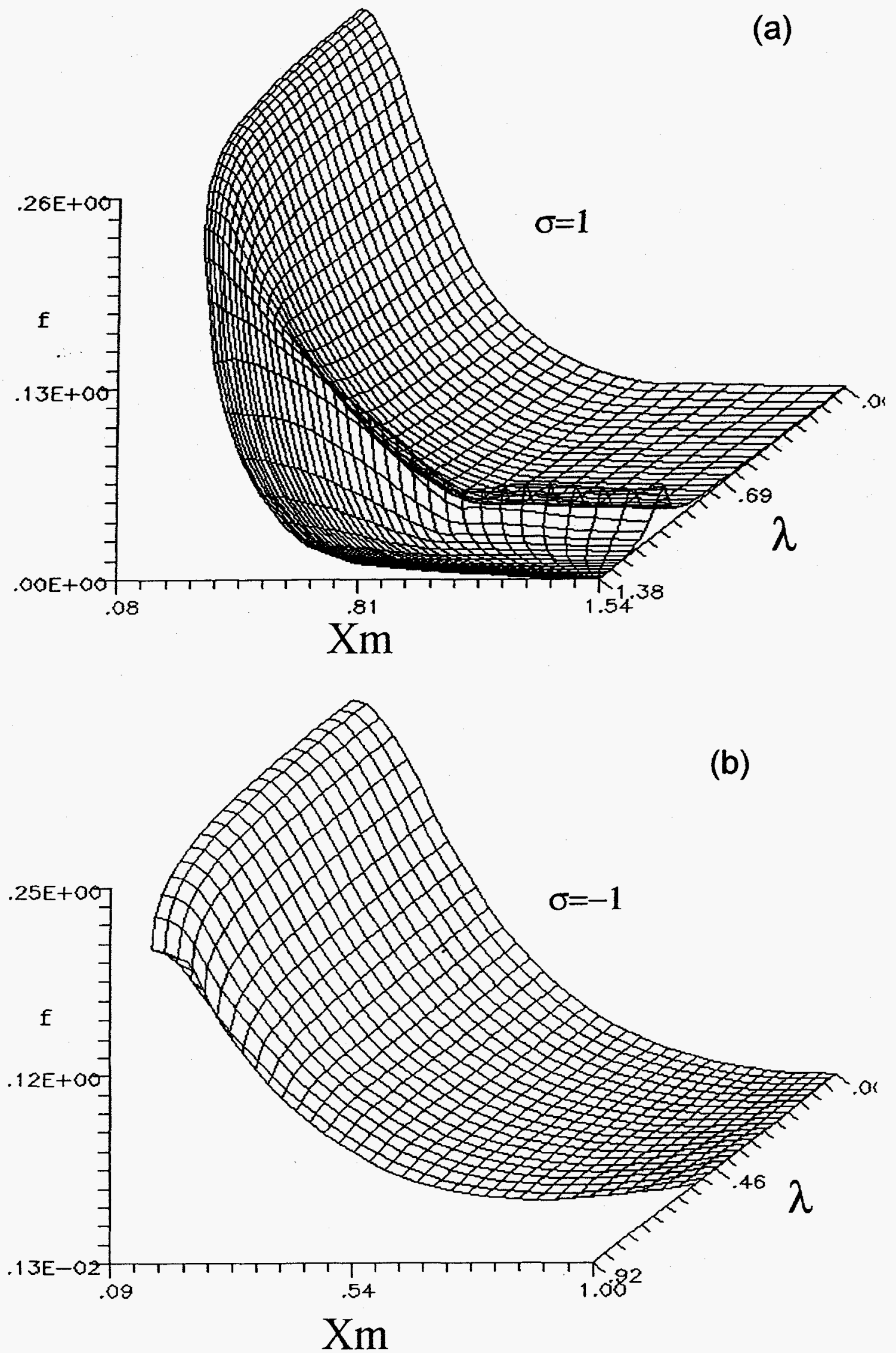

Fig. 5 

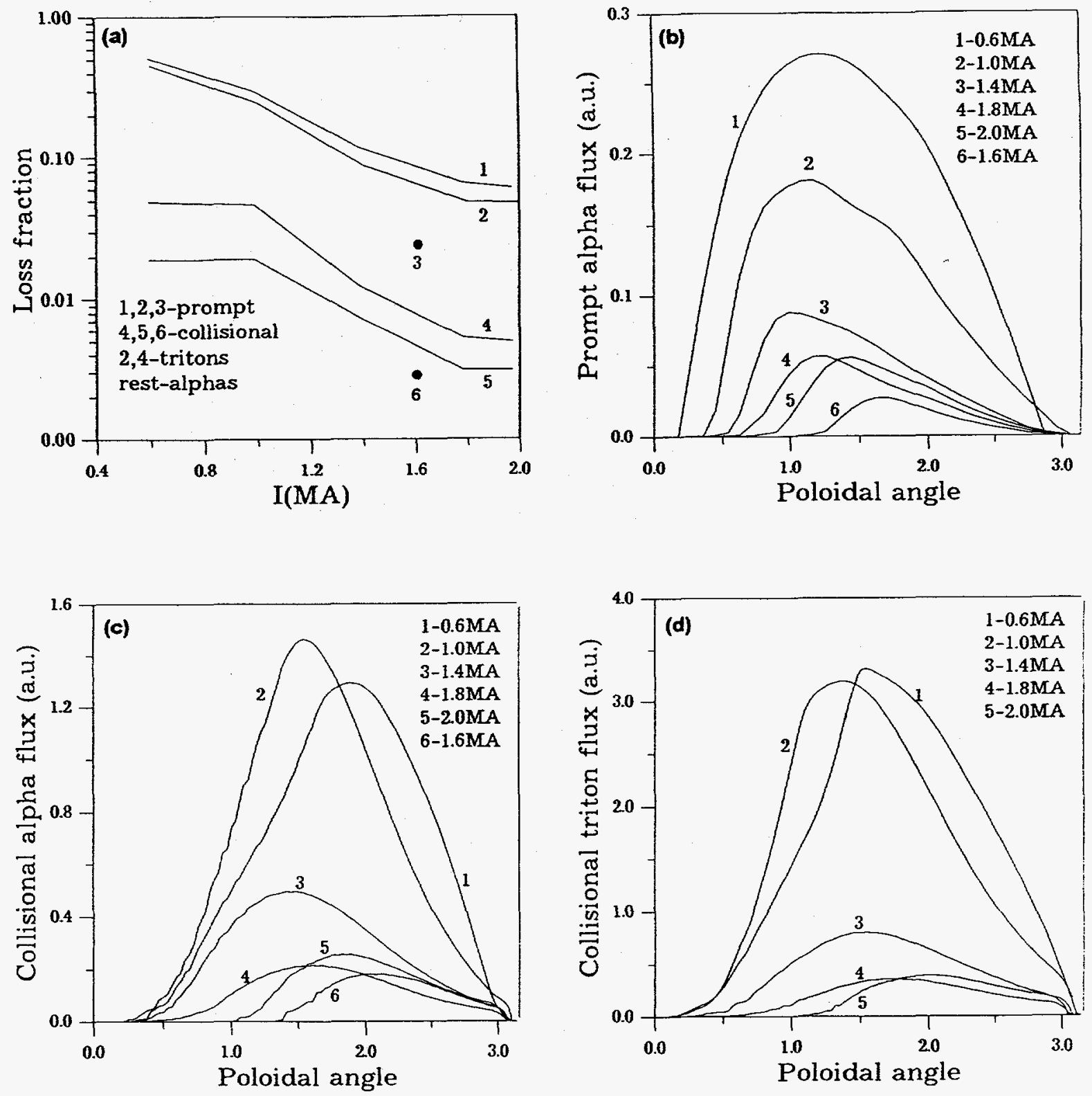

Fig.6 

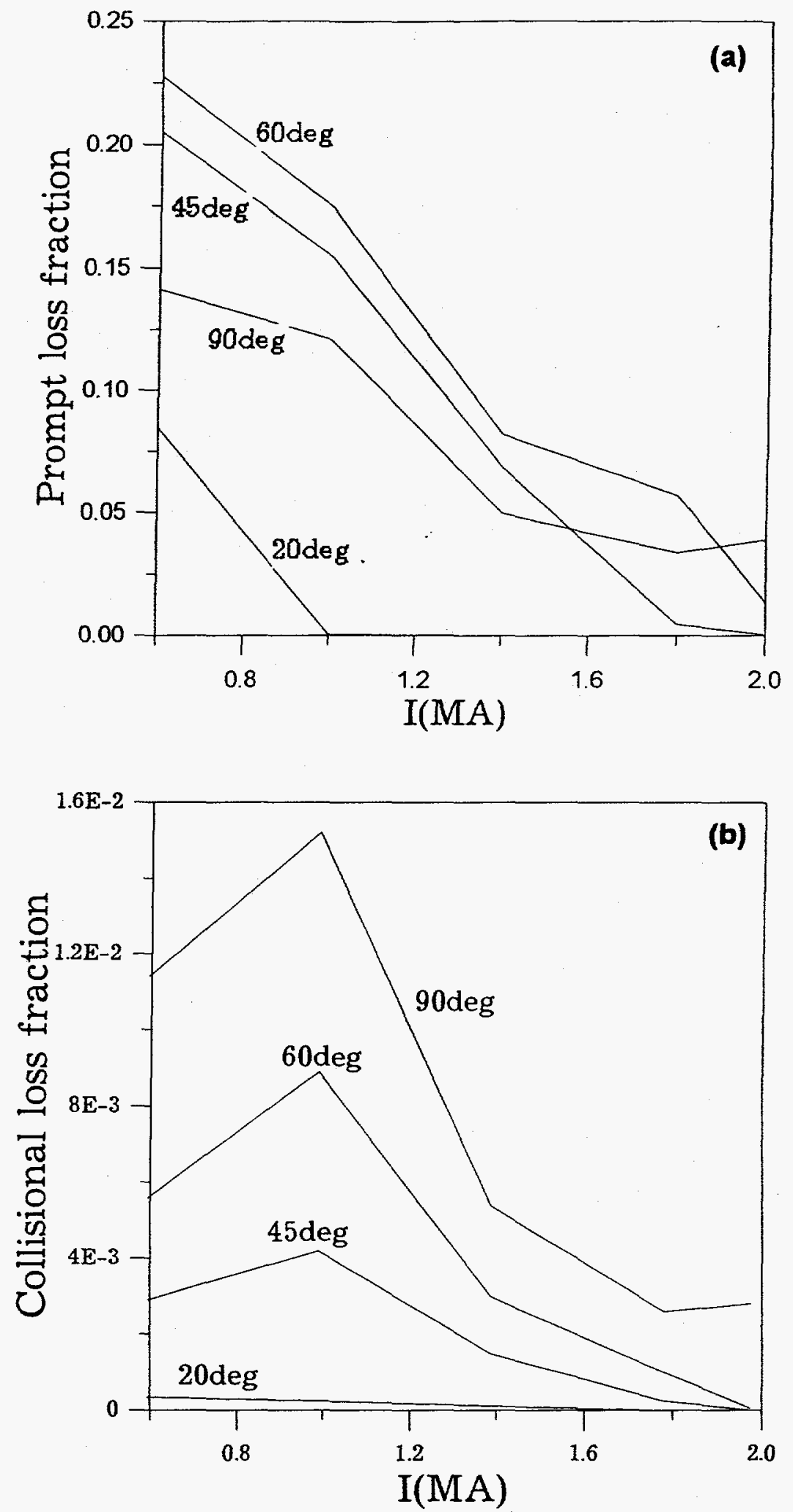

Fig.7 


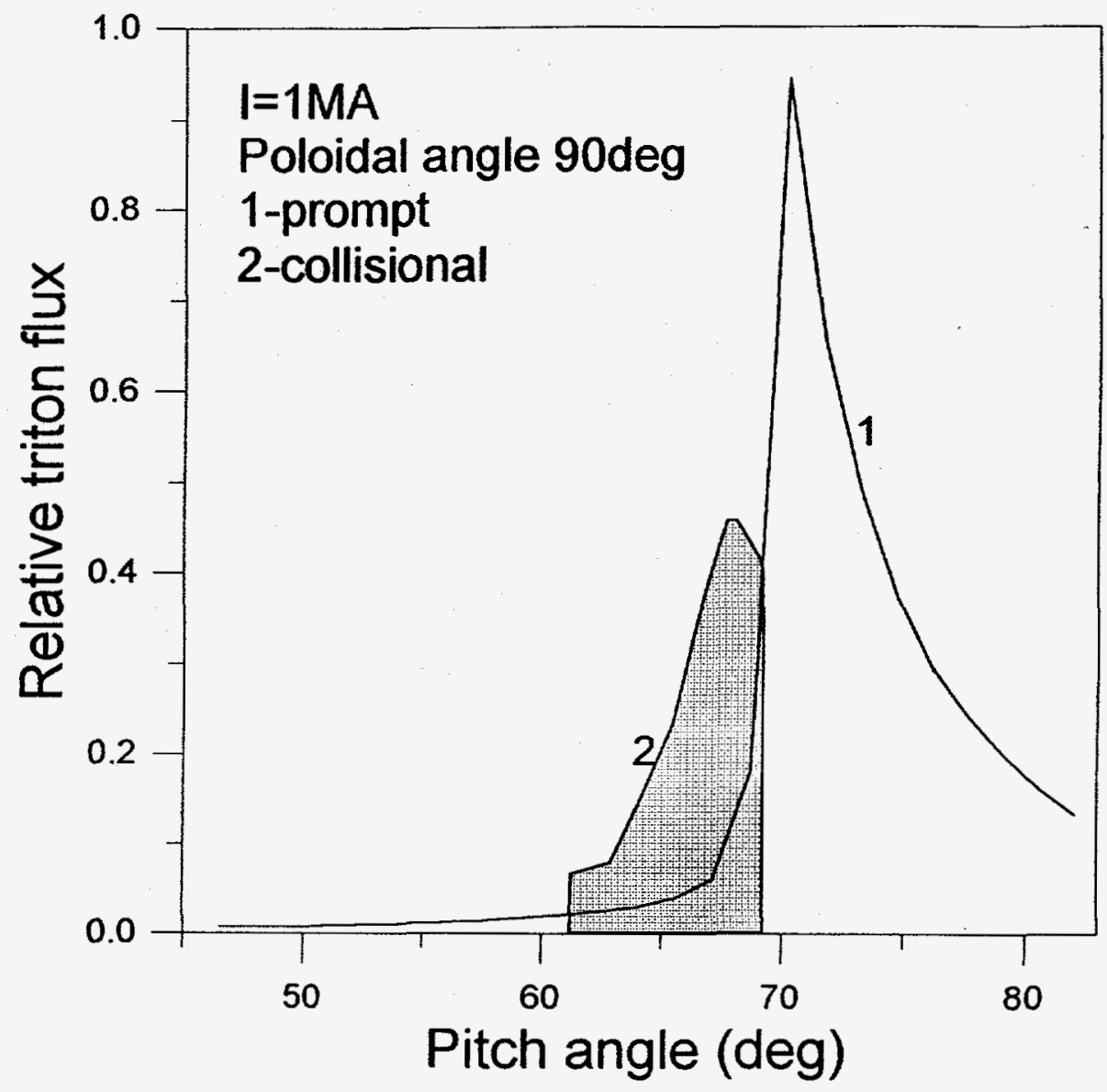

Fig. 8 

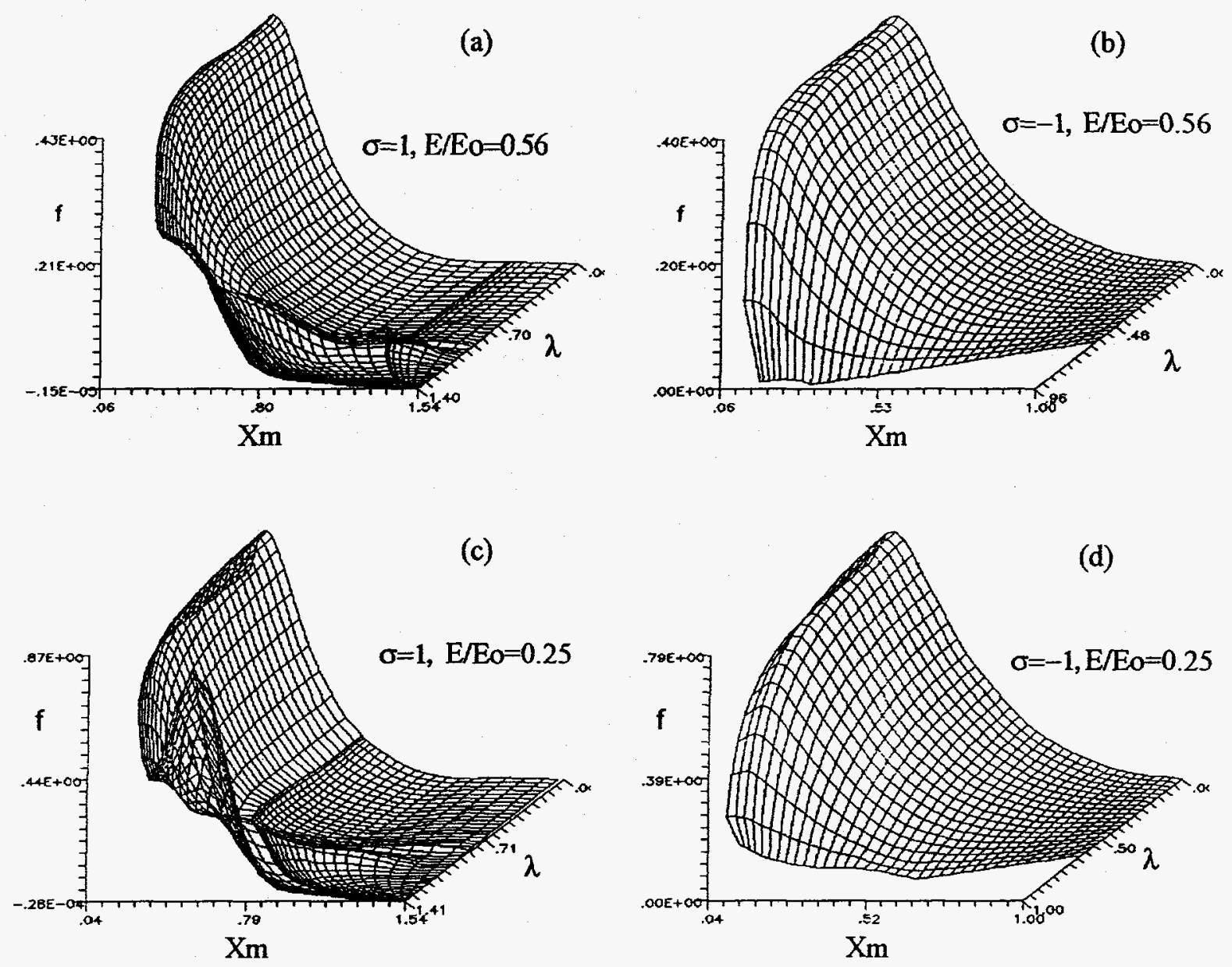

Fig.9 

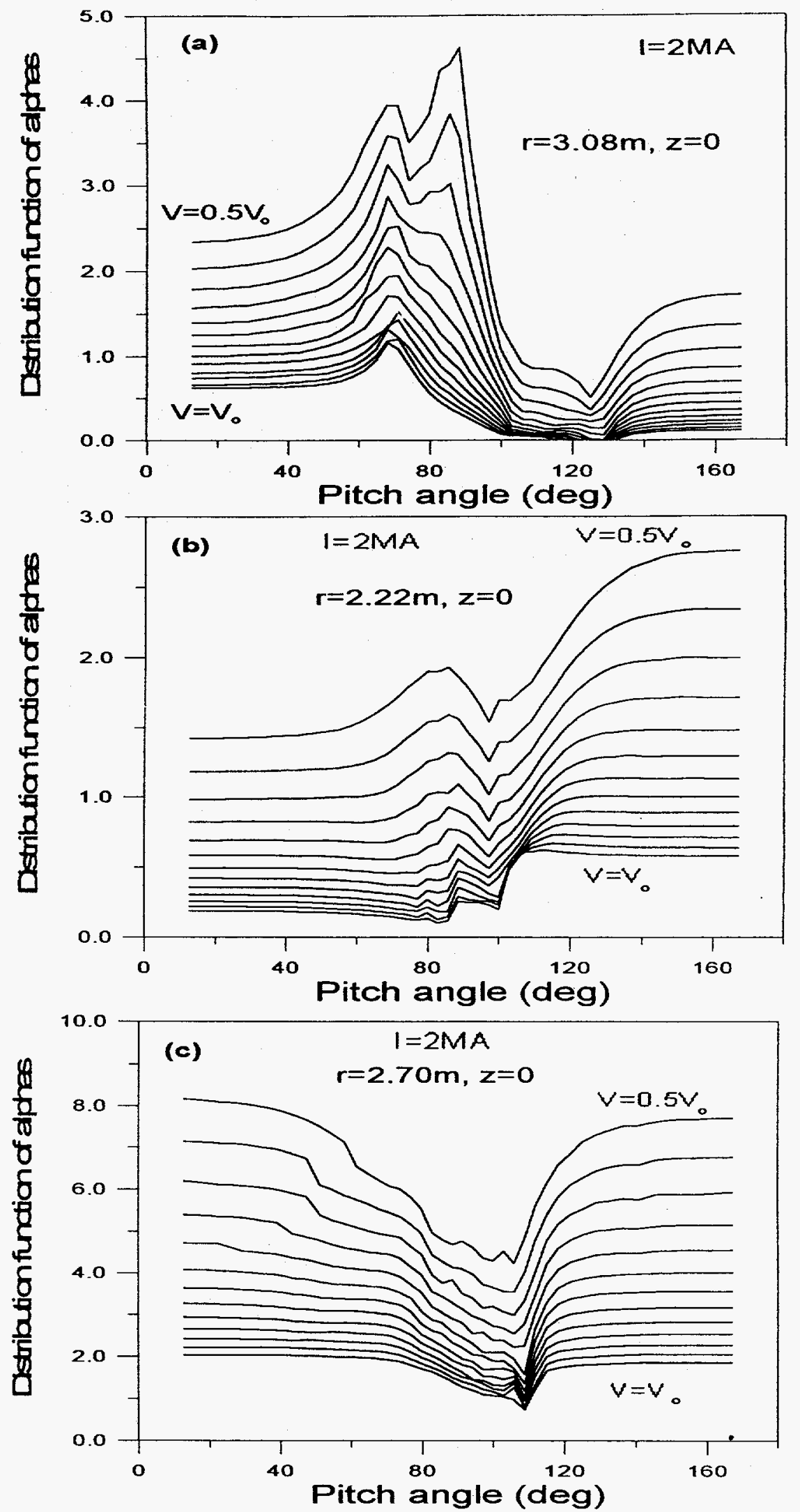

Fig. 10 

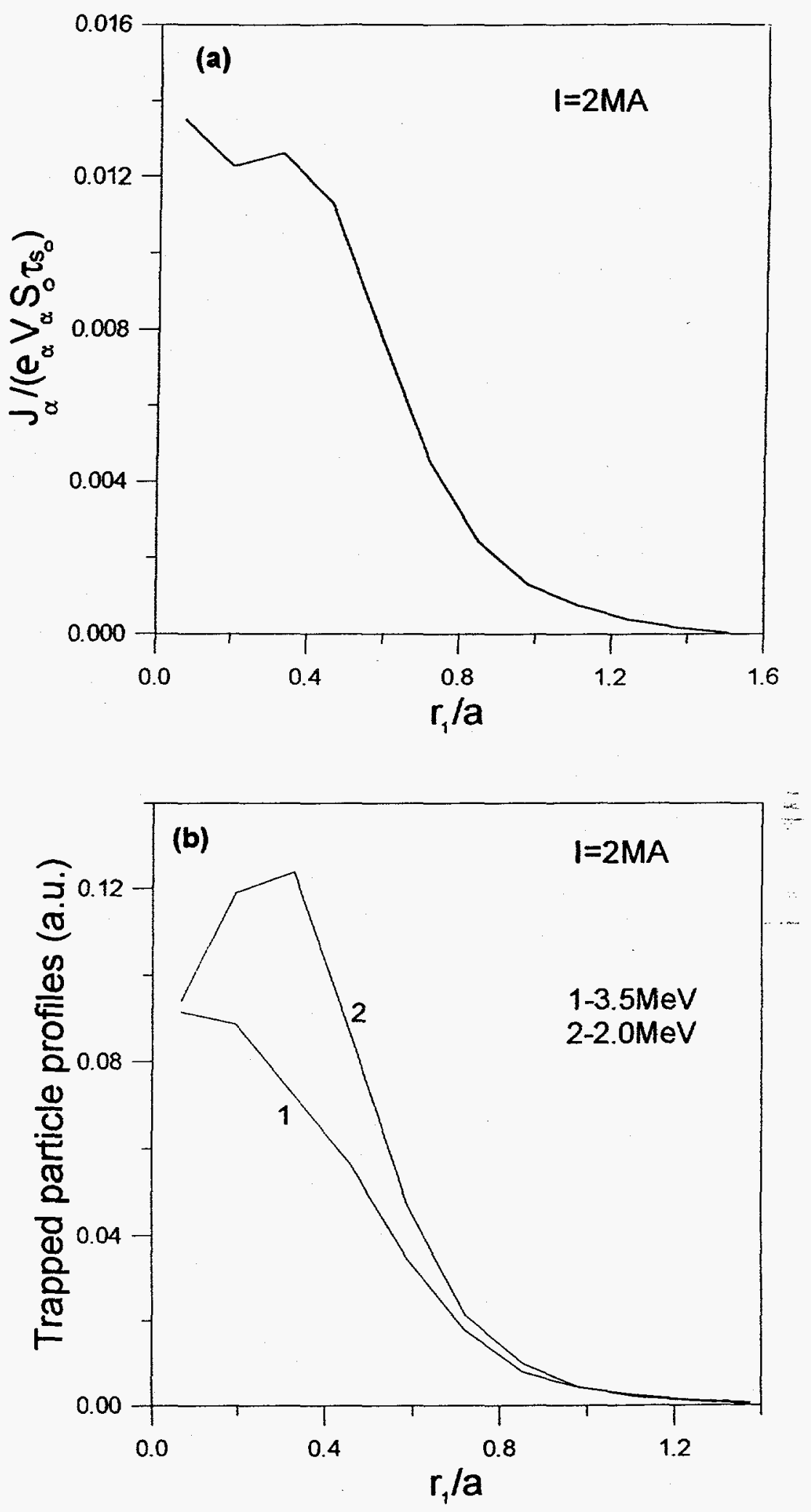

Fig.11 

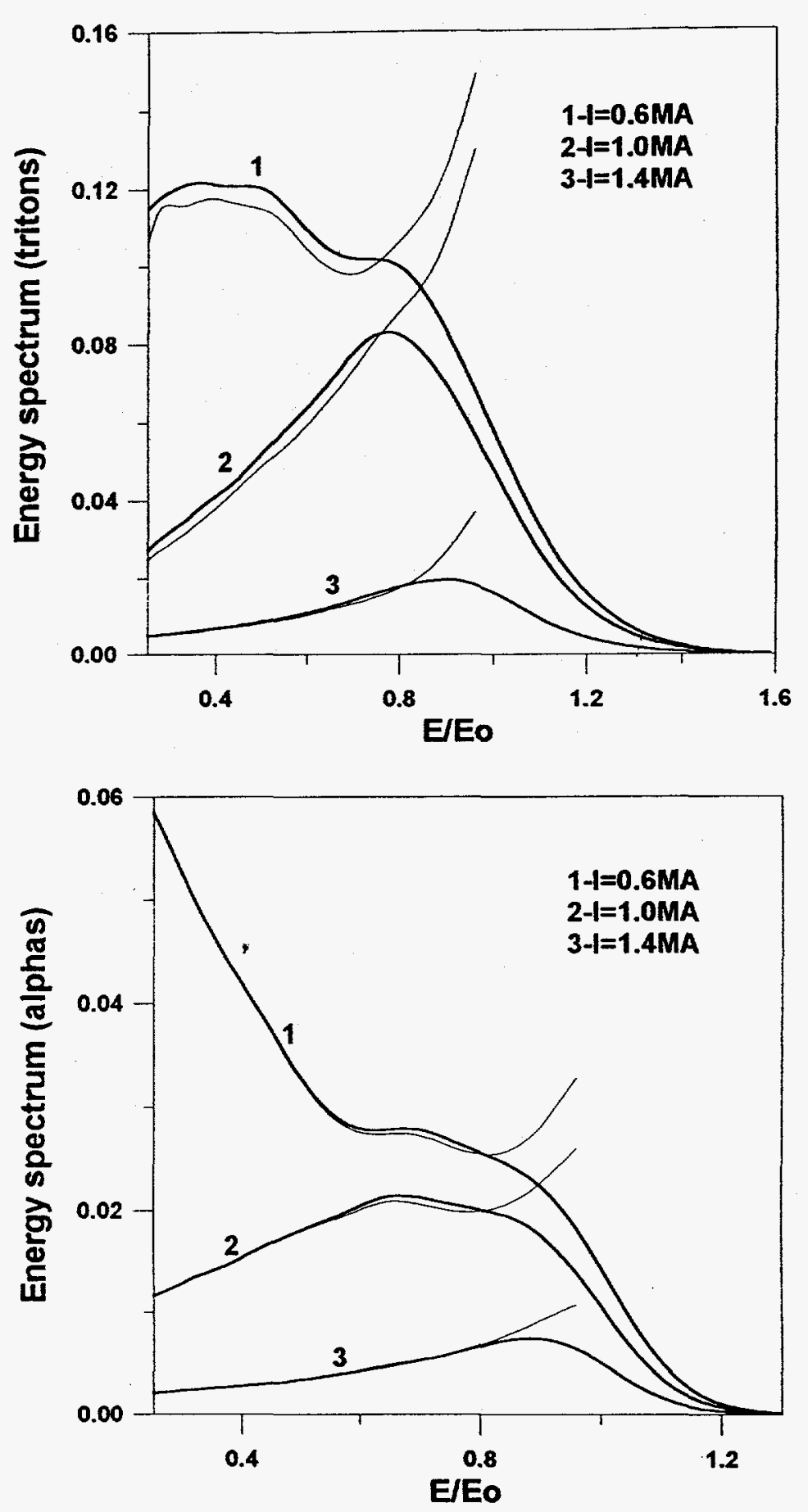

Fig. 12 